\section{Laryngoscope Tracheal Intuba- tion Education for Beginners Using a Smartphone and a Smartphone Endoscope}

\section{Sir,}

The process of intubation via direct laryngoscopy is complex and requires considerable technical skill. The success rate for a beginner is approximately $50 \%$. ${ }^{1}$ Further, a success rate of $90 \%$ cannot be expected until 50 attempts. ${ }^{2}$ The beginners working in the emergency department or intensive care unit, who are required to perform airway management frequently, require adequate training in laryngoscope intubation. The video laryngoscope is known for its efficacy in providing education of tracheal intubation. ${ }^{3}$ However, the obtained view is different from that obtained by an actual laryngoscope because it is built for accurate and safe tracheal intubation. As the obtained view is superior to that obtained by the laryngoscope, ${ }^{3}$ they are more suitable for teaching techniques for management of difficult cases and may not be suitable for education of a beginner for tracheal intubation usinglaryngoscopemanipulation. Further, videolaryngoscope is very expensive, and multiple units of the device in an educational institution might not always be available. We introduce an innovative method of education for learning techniques of laryngoscope tracheal intubation using a smartphone and an endoscope. The equipment used were: Mannequins for airway management: Laerdal $®$ Airway Management Trainer(Norway).

Laryngoscope with Macintosh No. 3 blade (INT; Intubrite LIC, Vista, CA, USA)

Smartphone (Galaxy S4, Samsung) and smartphone endoscope camera (Coms BE591, Lightcom, China).

Endotracheal tube and balloon syringe.

Camera mobile application (CameraFi, Vault Micro, Inc., Republic of Korea) The outer diameter of the endoscope was 5.5 $\mathrm{mm}$. It was fixed to the blade using a clip-on fixture. The light of the blade was removed to affix the endoscope. The lenses of the endoscope were located at a distance of $8.3 \mathrm{~cm}$ from the proximal end of the blade. The endoscope connects with the smartphone and activates the camera application. After the insertion of the laryngoscope, the supervisor evaluates its progress, as performed by the trainees, with a smartphone. The viewing angle shown on the smartphone with the endoscope optical lens was 44 degrees based on the junction of the lips and the blade. The location of the junction of the blade and the lip was $4.5 \mathrm{~cm}$ away from the proximal end of the blade toward the blade tip. The measured blade angulations, in the eye and the endoscope lens were 11 degrees. The principal blade angulations measured with the endoscope and the C-MAC video laryngoscope, are shown in Figure 1.

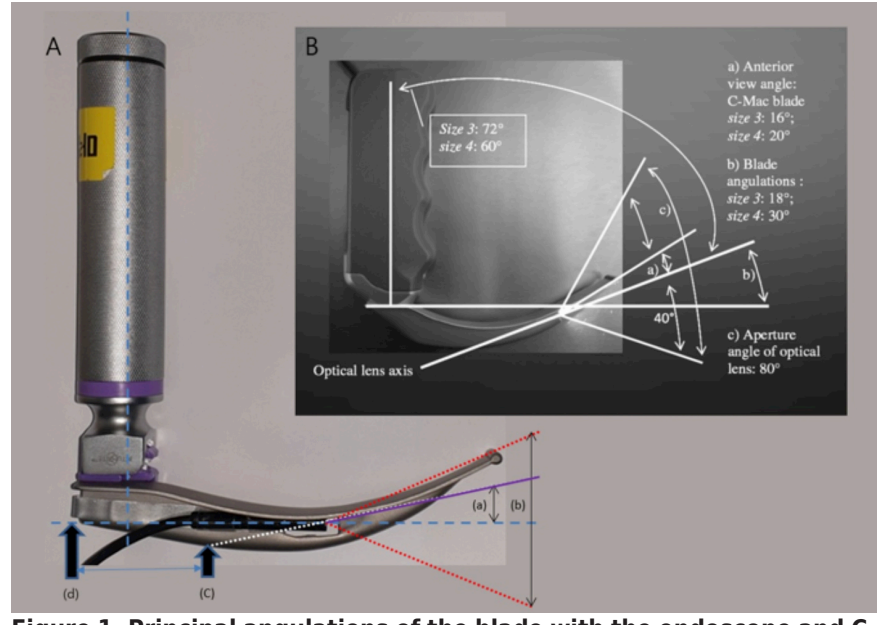

Figure 1. Principal angulations of the blade with the endoscope and CMAC videolaryngoscope:

(A) Laryngoscope with endoscope: (a) Anterior viewing angle of the endoscope and the eyes: $11^{\circ}$; (b) Viewing angle of the optical lens of the endoscope: $44^{\circ}$; (c) Contact between the blade and the lip: $4.5 \mathrm{~cm}$ away from the proximal end of the blade towards the blade tip; (d) The proximal end of the blade.

(B) C-MAC video laryngoscope (Cavus E, KieckhaeferJ, Doerges V, Moeller $T$, Thee C, Wagner K. The C-MAC video laryngoscope: First experiences with a new device for video laryngoscopy-guided intubation. Anesth Analg 2010; 110(2):473-7).

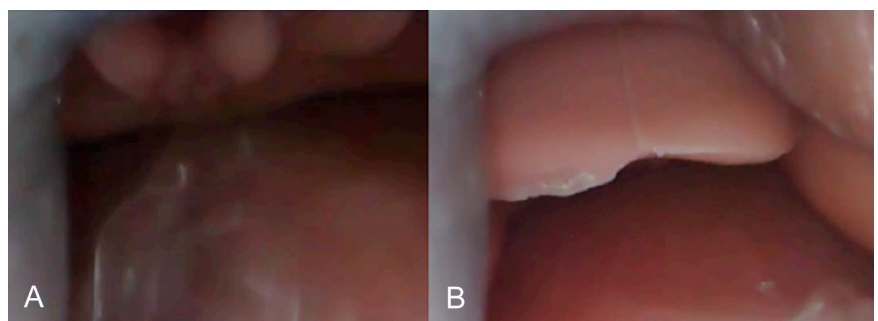

Figure 2. Endoscopic view of errors made by the trainees during laryngoscope insertion.

(A) The endoscopic view when blade insertion is deep. As the epiglottis has been lifted, only a part of the arytenoid cartilage is visible. (B) The endoscopic view when the laryngoscope is lifted insufficiently. The epiglottis is visible, but the glottis is not visible.

Ten medical students were trained using the above-elaborated equipment. Errors during tracheal intubation can be recorded as images. We were able to identify the mistakes that occurred during tracheal intubation (Figure 2). Previous study has shown the efficacy of tracheal intubation education by a video laryngoscope. The C-MAC has unique advantages of obtaining both a direct laryngoscopic view and a camera view that is displayed on the screen. However, the line-of-sight view of the glottis in the C-MAC video laryngoscope is better than the direct nakedeye view. ${ }^{3}$ The lens of the C-MAC is closer to the tip of the blade than the lens of the endoscope. As the point of viewing is closer to the glottis, a wider field of view is transmitted to the video monitor. The anterior viewing angle of the C-MAC was 16 degrees and was 5 degrees larger than that of the smartphone endoscope used in this study. light of the blade was removed to eliminate visual field obstruction caused by the endoscope during the use of the direct laryngoscope. Endoscopic light was used and its brightness was sufficient for tracheal intubation. If 
materials other than those used in this study are used, different measurements might be obtained. However, as shown in Figure 2 , if the position of contact of the blade and the lip is verified, a similar viewing angle of the laryngoscope and the endoscope can be obtained by adjusting the position of the endoscope.

As a smartphone is used, the portability is good. A smartphone endoscope to be fixed to the blade is inexpensive. In contrast, video laryngoscopes are less portable and expensive, depending on the equipment used. The current online price of video laryngoscopes is expensive.

Our device is not designed to provide a better view of the glottis than the direct laryngoscopic view. It is designed to make the endoscopic view similar to that of the actual laryngoscopic view, evaluation of the process of laryngoscope blade insertion is easier. The preferred use of this device is for supervision and guidance during tracheal intubation through direct laryngoscopy. The trainees can obtain a video of their course of tracheal intubation, and the video included the audio so that they could hear the supervisor's explanation of the wrong course of tracheal intubation.

\section{FUNDING:}

This research was supported by the First Research in Lifetime Program of the National Research Foundation (NRF) funded by the Korean government (MSIT) (NRF- 2018R1C1B5085866), South Korea.

\section{CONFLICT OF INTEREST:}

Authors declared no conflict of interest.

\section{AUTHORS' CONTRIBUTION:}

JWC, YSK: Drafted the work.

JHK: Drafted the work, and revised critically.
YSK: Contributed to the conception/design of the work and revised it critically.

\section{REFERENCES}

1. Levitan RM, Goldman TS, Bryan DA, Shofer F, Herlich A. Training with video imaging improves the initial intubation success rates of paramedic trainees in an operating room setting. Ann Emerg Med 2001; 37(1):46-50. doi: 10.1067/ mem.2001.111516.

2. Baker PA, Weller JM, Greenland KB, Riley RH, Merry AF. Education in airway management. Anaesthesia 2011; 66(s2):101-11. doi: 10.1111/j.1365-2044.2011.06939.x.

3. Cavus E, Kieckhaefer J, Doerges V, Moeller T, Thee C, Wagner K. The C-MAC videolaryngoscope: First experiences with a new device for videolaryngoscopy-guided intubation. Anesth Analg 2010; 110(2):473-7. doi: 10.1213/ANE.0b013 e3181c5bce5.

Jong Ho Kim ${ }^{1,2}$, Jun Woo Choi ${ }^{1}$ and Young Suk Kwon ${ }^{1,2}$

${ }^{1}$ Chuncheon Sacred Heart Hospital, College of Medicine, Hallym University, Chuncheon, South Korea

${ }^{2}$ Institute of New Frontier Research Team, Hallym University, Chuncheon, South Korea

Correspondence to: Dr. Young Suk Kwon, Department of Anaesthesiology and Pain Medicine, Chuncheon Sacred Heart Hospital, 77 Sakju-ro, Chuncheon, 24253, South Korea

E-mail: gettys@hallym.or.kr

Received: November 21, 2019; Revised: February 03, 2020; Accepted: February 11, 2020

DOI: https://doi.org/10.29271/jcpsp.2020.12.1365 